\title{
La regulación de las telecomunicaciones en Chile: El difícil camino hacia la transparencia
}

Chile fue el primer país de América Latina, y uno de los primeros en el mundo, en privatizar el área de las telecomunicaciones. Este país no cuenta aún, sin embargo, con un sistema regulatorio transparente. Si bien la Subsecretaría de Telecomunicaciones (hasta 1989, una institución más bien simbólica) recientemente ha comenzado a incluir a las empresas en las discusiones sobre reglas y normas técnicas, ésta aún no ha generado ningún espacio de participación para el público. Esto, junto con la exigencia de la Ley General de Telecomunicaciones de que el proceso tarifario se lleve a cabo en secreto, genera un clima de desconfianza que perjudica las inversiones en este sector. La autora de este artículo explica la necesidad de revertir esta situación a través de un sistema más transparente y participativo.

\section{Milagros Rivera Sánchez1}

Doctora en Comunicación por la Universidad de Florida. Profesora titular del departamento de Telecomunicaciones de la Universidad de Indiana y profesora visitante (2002-2003) del programa de Administración de Información y Comunicación de la Universidad Nacional de Singapur.

[mriveras@indiana.edu]

En 2002, la Ley General de Telecomunicaciones de Chile cumplió 20 años. Chile, pionero en la privatización de las telecomunicaciones, comenzó a impulsar ese sector hacia la competencia abierta e indiscriminada en 1982.2 Dos décadas más tarde, muchos países están aún moviéndose en esa dirección, algunos con más éxito que otros. De hecho, de acuerdo con la Unión Internacional de Telecomunicaciones, la competencia abierta en ese mercado existe sólo en 16 países (Tabla 1). Chile es uno de ellos.

Tres componentes importantes en la reforma de las telecomunicaciones son: la privatización de la industria, la introducción de competencia y la creación de un ente regulador independiente y experto. 3 Este último componente, en particular, aumenta la posibilidad de que se establezcan reglas y leyes que promuevan una competencia justa entre los proveedores de servicios de telecomunicaciones.

De acuerdo con investigadores de políticas reguladoras, es importante que el sector en consideración perciba que las reglas del juego son justas. De lo contrario, las empresas pueden perder confianza en el regulador y reevaluar sus inversiones. 4 La regulación de las telecomunicaciones, por lo tanto, puede impactar de manera significativa la economía de un país. En muchos casos, el regulador actúa como árbitro, balanceando los intereses de las empresas dominantes con los de las empresas que están tratando de entrar al mercado. 5

El problema en muchos países en desarrollo es que tienen poca experiencia en la regulación de este sector. Como el movimiento global hacia la privatización de las telecomunicaciones se ha dado sólo en los últimos diez años, la gran mayoría de los entes reguladores que existen hoy día son relativamente nuevos. (Tabla 2.) El de Chile fue el primero en América Latina y uno de los primeros del mundo. Debe tomarse en cuenta, sin embargo, que durante el gobierno de Augusto Pinochet, el rol de la Subsecretaría de Telecomunicaciones (Subtel) era más simbólico que real ya que el subsecretario era un militar con rango de coronel mientras que los dirigentes de las compañías telefónicas CTC y Entel (Compañía de Telecomunicaciones de Chile y Empresa Nacional de Telecomunicaciones, respectivamente) eran militares con rango de general. No se puede decir con seriedad que el oficial subalterno haya tenido poder alguno para supervisar las acciones de las empresas reguladas.

Pero aunque el poder del regulador fuera ilusorio, es posible argumentar que la infraestructura para regular el sector de las telecomunicaciones en Chile se estableció con la creación de la Subtel. De hecho, este fenómeno de un regulador novato que es percibido como ineficiente o débil no es raro. Muchos países comienzan a regular sus empresas de 
telecomunicaciones de forma tambaleante, hasta que se dan cuenta de que la única forma de atraer (y retener) capital extranjero es con reglas consistentes y un sistema regulatorio transparente. 6

El objetivo de este estudio es determinar cuán transparente es el proceso regulatorio de las telecomunicaciones en Chile y cómo la Subtel interactúa con el sector regulado y el público. Para obtener una visión realista de estas interacciones y del proceso regulatorio, entrevisté a funcionarios y ex funcionarios de la Subtel, ejecutivos de las empresas del sector, dirigentes de grupos ciudadanos y académicos y expertos en el ámbito de las telecomunicaciones.

\section{Transparencia en la regulación: ¿realidad o meta esquiva?}

«Transparencia» es la palabra que escuché con más frecuencia en mis conversaciones con funcionarios de la Subtel. En Chile, sin embargo, la transparencia en el ámbito regulatorio de las telecomunicaciones todavía no tiene el significado que los investigadores académicos le asignan a ese término. De acuerdo con Frieden, la transparencia regulatoria implica cuatro elementos. Primero, el público debe tener oportunidad de participar en el proceso regulatorio, ya sea por escrito o a través de reuniones públicas. Segundo, la agencia reguladora debe crear un expediente que todas las partes interesadas puedan examinar y evaluar. Tercero, el proceso regulatorio debe ser justo y seguir reglas que se apliquen de forma consistente. Finalmente, la agencia reguladora debe explicar en qué información basó sus decisiones (o las normas o reglas que creó).10 Idealmente, esas explicaciones (o directrices) debieran publicarse para que todos los interesados puedan examinarlas. 11

Otros académicos dividen el concepto de transparencia en dos: transparencia informativa y transparencia participativa.12 La primera no sólo implica que el público tenga acceso a información sobre las acciones y decisiones de los actores gubernamentales, sino también aquella con la cual se toman esas decisiones. La transparencia informativa se puede lograr cuando el gobierno emite directrices explicando el porqué de sus acciones. Transparencia participativa implica participación pública en decisiones políticas, ya sea en forma representativa -por ejemplo, a través de grupos ciudadanoso vía participación directa.

Un grupo de investigadores señala que la transparencia en las acciones gubernamentales es la característica que más claramente define a las democracias modernas.13 Demchak, Friis y La Porte aseguran que los gobernantes son más efectivos y honestos cuando sus acciones están sujetas al escrutinio del pueblo y cuando los ciudadanos tienen acceso a la información que el gobierno utiliza para realizar sus funciones.

Aunque la transparencia parece ser una meta importante en la administración del Presidente Ricardo Lagos, se requieren varios cambios fundamentales para que realmente exista transparencia reguladora en Chile. Por ejemplo, las agencias reguladoras tienen que cambiar su forma de operar, los funcionarios públicos tienen que considerar el concepto de participación pública en forma más amplia y las leyes que promueven discusiones de carácter secreto deben ser eliminadas. De lo contrario, el concepto de transparencia es una palabra hueca, que se repite porque políticamente suena bien. Por supuesto, estos cambios toman tiempo y no siempre son fáciles de implementar, pero no hay duda de que una vez que el proceso de apertura y transparencia comienza, es difícil dar marcha atrás.

La Subtel ha hecho esfuerzos loables por incluir a las empresas de telecomunicaciones en las discusiones sobre reglas y normas técnicas. Sin embargo, el público no está nunca invitado a participar -formal o informalmente- en ninguno de los procesos de la agencia, incluyendo el de fijación de tarifas. 14 Por otro lado, mientras la Subtel trata de generar un ambiente de cooperación con los regulados, la Ley General de Telecomunicaciones requiere que el proceso tarifario se lleve a cabo en secreto. Esto crea un clima de desconfianza contra la agencia y el Ministerio de Obras Públicas, Transportes y Telecomunicaciones, especialmente cuando empresas reguladas, como es el caso de la CTC, cuestionan la validez del decreto tarifario. Continuar con un sistema tan cerrado cuando la política de la administración Lagos está abogando por un gobierno transparente equivale a mantener la puerta cerrada mientras se invita a alguien a que entre por el ojo de la cerradura. 
Varios expertos en política pública definen el proceso regulador como uno de negociación, donde el poder relativo y la influencia de los grupos interesados en participar afectan los resultados.15 Otros expertos han creado modelos en que se identifican los principales actores y se trata de anticipar su efecto en el proceso regulador.16 En los Estados Unidos, por ejemplo, los actores principales, de acuerdo con Krasnow, Longley y Terry, son el Congreso, el Presidente, las cortes, la Comisión Federal de Comunicaciones (FCC), las empresas reguladas y el público. En Chile, el elenco de actores es similar. El Presidente nombra al Ministro de Obras Públicas, Transportes y Telecomunicaciones y al Subsecretario de Telecomunicaciones. El Congreso pasa las leyes y las cortes resuelven disputas que no se pueden resolver por la vía administrativa. La Subtel está a cargo de otorgar concesiones y redactar reglas y normas técnicas. Asimismo, junto al Ministerio de Obras Públicas, Transportes y Telecomunicaciones, la Subtel regula las tarifas de empresas que carecen de competencia (como la CTC).

\section{Participación del Sector Regulado}

A pesar de que la diferencia más obvia entre el modelo regulador de los Estados Unidos y el de Chile es la ausencia total, en este último país, de la participación del público,17 las empresas reguladas sólo comenzaron a ser actores importantes hace poco. Hasta el año 2000, la Subtel creaba reglas y normas técnicas sin consultar a las empresas de telecomunicaciones. 18 Pero en ese año la agencia creó dos mecanismos para aumentar la transparencia. El primero estableció como política interna invitar a las empresas a participar en discusiones, con los funcionarios de la Subsecretaría, sobre el establecimiento de nuevas reglas. El segundo fue la creación del Comité Consultivo (CC) de las Telecomunicaciones.

El CC está compuesto por representantes de las empresas de telecomunicaciones que operan en Chile -proveedores de equipos, operadores y proveedores de servicio de internet- quienes ayudan a la Subtel a conseguir información y a redactar los borradores de nuevas reglas y normas técnicas. Algunos de los miembros del CC pueden ser llamados a participar en grupos de trabajo, como son el de televisión digital y el de internet de tercera generación. La composición de estos grupos depende del tema en cuestión, pero, de acuerdo con un miembro del CC, la Subtel permite que participen los involucrados en el sector regulado que manifiestan su interés por hacerlo.

Aunque la petición de un estudio puede originarse en la agencia misma o en las empresas reguladas, la Subtel es la que crea todos los grupos de trabajo. Éstos tienden a ocuparse de asuntos técnicos, administrativos u operacionales. Las reuniones, con excepción de la primera, se llevan a cabo a través de internet.

La participación de los grupos de trabajo se divide generalmente en dos etapas. En la primera, se recopila información técnica y se investiga cómo se están manejando en otros países los asuntos relacionados con la tecnología que compete al grupo. El informe final -que debe ser entregado a la Subtel en la fecha previamente determinada por ésta- generalmente incluye información básica sobre la tecnología en cuestión, el marco regulador, el equipo disponible y las tendencias del mercado. En la segunda etapa, la Subtel escoge un nuevo grupo de trabajo que se ocupa de redactar el borrador de la regla o norma técnica. En cada uno de estos grupos participa un funcionario de Subtel.

Durante el proceso de redacción de las reglas o normas técnicas, miembros del sector regulado pueden acercarse a oficiales de la Subtel y hacer recomendaciones o expresar sus preocupaciones. Sin embargo, un integrante del CC indicó que los miembros de este comité tienen como regla interna no usar las reuniones de éste para tratar de influenciar a la Subtel.

Luego de entrevistar a varios miembros del Comité Consultivo, es claro que todos están conscientes de que su rol es asesorar a la Subtel. Pese a que valoran la oportunidad de participar en este proceso de redacción del borrador de regulaciones, los entrevistados reconocieron que es la Subsecretaría la que determina qué estipulaciones van a aparecer en las reglas o normas técnicas. 
Uno de los miembros del CC indicó que participar en el comité tiene un beneficio indirecto, pero igualmente importante: Todos los competidores del sector están obligados a dialogar entre sí. Al final, cuando se redacta el borrador de una norma o regla, aunque no todos los miembros del grupo de trabajo estén satisfechos con el resultado, cada uno reconoce que tuvo la oportunidad de participar en el proceso, dice uno de los integrantes del Comité. Otros incluso creen que por generar la oportunidad de exponer sus preocupaciones ante la Subtel, el sistema de grupos de trabajo previene, en cierto grado, que las empresas que han integrado estos grupos sientan la urgencia de protestar ante los tribunales por las normas creadas. No es posible documentar si esto es cierto, pero miembros del CC coincidieron en que una ventaja definitiva de los grupos de trabajo es que se ha acortado el tiempo para sacar las normas y reglas. En el pasado, el trabajo de investigación y redacción de una norma técnica o una regla podían tomar más de un año. Ese tiempo se ha acortado a seis u ocho meses, lo cual puede tener un impacto significativo en la introducción de nuevos servicios. Juanita Gana, ex Subsecretaria de Telecomunica-ciones, explica que aunque la Subtel hace un esfuerzo por hacer el proceso regulador más transparente y participativo, la ley no lo requiere. La agencia lo está haciendo por iniciativa propia, lo cual significa que bajo una administración diferente, podría volver a su práctica de actuar en forma unilateral, sin solicitar la participación del sector regulado.

\section{Hacia un sistema regulador transparente}

Aunque la inclusión de las empresas de telecomunicaciones en el proceso regulador es un avance importante, aún queda mucho por hacer para alcanzar verdadera transparencia en esta materia. Los próximos pasos deben ser: (1) incorporar participación ciudadana y (2) crear un sistema que permita al público, y a otras partes interesadas, tener acceso a los procesos de la agencia y a los documentos utilizados durante esos procesos.19

•Participación ciudadana

En 1999, la Subtel comisionó un estudio con Participa, una corporación privada que promueve la participación ciudadana. Participa examinó el proceso de participación ciudadana en la regulación de las telecomunicaciones en Argentina, Canadá y Estados Unidos. Basado en la forma en que esos países involucraban a los ciudadanos en sus procesos de regulación, el estudio incluyó la propuesta de una enmienda a la Ley General de Telecomunicaciones para permitir distintos grados de participación de los ciudadanos en el proceso tarifario, desde tener acceso a información (algo que aún no sucede en Chile) hasta involucrarse más activamente en el proceso y hacer recomendaciones. El mayor obstáculo en este sentido en el área de las telecomunicaciones es que los temas técnicos y económicos que se discuten en el proceso de fijación de tarifas -y en la creación de normas técnicas, por ejemplo- son sumamente complejos. De ahí que el estudio de Participa recomendase que la empresa sujeta a la fijación de tarifas financie a un experto que represente los intereses de los ciudadanos y trabaje directamente con éstos. El estudio fue presentado a Subtel, pero luego de las elecciones y el subsiguiente cambio de gobierno en 2000 , nadie parece saber qué sucedió con él.

\section{-Acceso a información}

Las recomendaciones de Participa pudieron haber sido el primer paso de Chile hacia la transparencia reguladora en las telecomunicaciones; al menos en lo referente a la fijación de tarifas. Pero es importante recalcar que una participación efectiva requiere acceso a información y ése es tal vez uno de los problemas principales con la ley actual. Por ejemplo, el proceso tarifario -que es sólo parte del problema- es totalmente cerrado, sin posibilidad alguna de escrutinio público. Por lo tanto, la Subtel debe cambiar la forma en que define «participación» y «transparencia.»

«Participación» debiera incluir no sólo a las empresas reguladas sino también a miembros del público y de la comunidad académica, entre otros. Desde perspectivas distintas de la sociedad chilena ellos pueden hacer contribuciones importantes al proceso regulador. Sus comentarios y sugerencias podrían ser publicados en el sitio online de la Subtel de 
modo que todas las partes interesadas pudiesen evaluarlos y responder. Este intercambio de ideas movería a la Subsecretaría hacia un sistema de regulaciones más transparente y participativo y podría promover ideas innovadoras que beneficiarían al público y a las empresas de telecomunicaciones.

\section{Reformas al Sistema Regulatorio}

Aunque en mis entrevistas con funcionarios de la Subtel no percibí ninguna urgencia de incluir al público en los procesos de la agencia,20 no hay duda de que éste es el momento oportuno para hacer cambios legislativos y regulatorios que promuevan la transparencia. La Subtel comenzó a moverse en esa dirección en 2000 y en 2001 inició un proceso de auto evaluación que incluye un examen crítico de la Ley General de Telecomunicaciones. A través de reuniones con representantes de las empresas de telecomunicaciones, ex oficiales de la Subsecretaría y ex ministros de Obras Públicas, Transportes y Telecomunicaciones, expertos en el campo de las telecomunicaciones y representantes de los intereses ciudadanos; la agencia espera recoger recomendaciones que serán incorporadas en un «libro blanco.» Éste sería la base para determinar qué cambios son necesarios para promover eficiencia y transparencia. 21 Idealmente, la Subtel también debería usar esta oportunidad para incorporar mayor participación pública en el proceso regulador. Uno debe recordar que nadie -ni los funcionarios de la Subtel, ni los representantes de las empresas reguladastiene el monopolio de las buenas ideas. Ésta es la oportunidad de la Subtel para demostrar que en sus veinte años ha alcanzado un punto de madurez tal que la participación pública -incluyendo ciudadanos individuales, grupos representantes, profesores $\mathrm{u}$ otros expertos- se puede convertir en una realidad.

No hay duda de que el sector de las telecomunicaciones de Chile ya ha dado los primeros pasos hacia un sistema regulador más transparente y participativo. Todos los empresarios que entrevisté evaluaron a la Subtel y sus esfuerzos en ese sentido muy favorablemente. Esto indica que la agencia y sus funcionarios son capaces de implementar cambios de forma rápida y eficiente. Sin embargo, sería bueno que el proceso de participación que la Subtel creó en el 2000 (por ejemplo, el Comité Consultivo) fuera codificado formalmente. Eso evitaría que los logros alcanzados hasta ahora se perdieran en administraciones futuras.

\section{Notas}

1. La autora realizó la investigacion para este artículo durante su permanencia de tres meses en Santiago, Chile, con una beca de investigación de la Comisión Fulbright. Desea expresar su agradecimiento a esa institución en Chile y en Estados Unidos y a las siguientes personas y organizaciones: Indiana University; Patricio Bernedo, Eliana Rozas y Soledad Puente, de la Facultad de Comunicaciones la Pontificia Universidad Católica (UC); Fanor Larraín Verdugo, del Instituto de Ciencia Política, UC; Ángela Vivanco, Facultad de Derecho, UC; Mirtha Campos, de El Mercurio; Ricardo Paredes, de la Universidad de Chile; Alberto Cabezas, de la Red Universitaria Nacional; José Ricardo Melo, consultor; Renato Jijena Leiva, abogado y consultor; Rossella Cominetti, Edgardo Mímica, Leonardo Mena, Jaime Prado, y Juan Luis Pérez, de la Subtel; Francys Lillo, de Motorola; Rodolfo Herrera Bravo, de la Contraloría General de la República; Juanita Gana; miembros de la Corporación Nacional de Consumidores y Usuarios (CONADECU), especialmente Ernesto Benado; Consumers International; Andrea Sanhueza, de Participa; y a numerosos ejecutivos de las empresas de telecomunicaciones de Chile.

2. La privatización de CTC y Entel comenzó en el 1982 pero no se completó hasta 1990. Ver: Melo, José Ricardo (1997): «Regulación de Telecomunicaciones en Chile, Perú y Venezuela,»Instituto Latino Americano de Planificación y Economía Social y Corporación Andina de Fomento; Melo, José Ricardo (1998): Chile. En Noam, E. M. (Ed.), Telecommunications in Latin America, pp. 202-226.

3. Wallsten, Scott J. (1999, mayo): An Empirical Analysis of Competition, Privatization, and Regulation in Africa and Latin America. En World Bank: Infrastructure. Documento de trabajo. Ver también Frieden, Rob (2001): Managing InternetDriven Change in International Telecommunications, pp. 86-89. 
4. Fink, Carsten; Mattoo, Aaditya; y Rathindran, Randeep (2001, nov.): Liberalizing Basic Telecommunications: The Asian Experience. En World Bank: Infrastructure. Documento de trabajo 2718.

5. Frieden, R.: op. cit., pp. 85-87.

6. Ver, por ejemplo, Freempong, G.K. y Atubra, W.H. (2001): Liberalisation of Telecoms: The Ghanaian Experience, Telecommunications Policy, 25, pp. 197-210.

7. Unión Internacional de Telecomunicaciones, www.itu.org (Sitio visitado el 10 de febrero de 2002).

8. Japón es un caso excepcional porque, a pesar de tener un sistema de competencia abierta, no cuenta con un ente regulador independiente.

9. Hay otros países que crearon un ente regulador en la década de los noventa (tal como Argentina, Hungría, Letonia y Venezuela), pero la Unión Internacional de Telecomunicaciones ha determinado que en esos paises los reguladores no son independientes de las fuerzas políticas. Unión Internacional de Telecomunicaciones, www.itu.org (visitada el 15 de febrero de 2002).

10. Frieden, R.: op. cit.

11. Ver: Galetovic, Alexander y Sanhueza, Ricardo (2002, verano): Regulación de Servicios Públicos: ¿Hacia dónde debemos ir? En Revista Estudios Públicos. (El artículo no había sido publicado en el momento en que se escribió este artículo, pero la autora tuvo acceso a él a través de una copia que le envió uno de los autores vía correo electrónico).

12. Balkin, Jack M.: How Mass Media Simulate Political Transparency, Yale University: Writings Online, www.yale.edu/lawweb/jbalkin/articles/media01.htm. (Sitio visitado el 20 de febrero de 2002.)

13. Demchak, Chris C; Friis , Christian; y La Porte, Todd (2000) : Webbing Governance: National Differences in Constructing the Face of Public Organizations, en Garson, G. David (Eds.): Handbook 014. La ley no le provee a Subtel la discreción de abrir el proceso.

15. Bitran, E., y Serra, P. (1998): Regulation of privatized utilities: The Chilean experience, World Development, 26(6), pp. 945-962; Sinha, N. (1995): Regulatory reform: an institutional perspective. En Mody, B., Bauer, J. M. y Straubhaar, J. D. (Eds.): Telecommunications Politics: Ownership and Control of the Information Highway in Developing Countries, pp. 287-308.

16. Krasnow, Longley y Terry (1982): The Politics of Broadcast Regulation, p.1.

17. El propósito de este estudio no es determinar si el público tiene influencia significativa en el proceso regulatorio. Ésa es una pregunta importante, pero que no podría ser contestada aquí.

18. Entrevistas con: Alberto Cabezas, gerente de comunicaciones, Red Universitaria Nacional (29 de mayo de 2001); Juanita Gana, ex subsecretaria de Telecomunicaciones (23 de mayo de 2001); Francys Lillo, gerente de relaciones gubernamentales de Motorola (11 de mayo de 2001); Leonardo Mena, jefe de la oficina de infraestructura para internet de la Subtel (24 de mayo de 2001).f Public Information Systems, Nueva York: Marcel Dekker.

19. En caso de que alguien

piense que mi análisis está sesgado por mis experiencias en los Estados Unidos, sugiero que lean el artículo escrito por los profesores chilenos Alexander Galetovic y Ricardo Sanhueza de la Universidad de Chile: «Regulación de Servicios Públicos: ¿Hacia Donde Debemos Ir?» Se sugiere allí que en Chile debe aumentar la transparencia regulatoria. También recomiendan que las agencias reguladoras debieran crear un sistema para acopiar información relacionada con el proceso tarifario y proveer acceso al público a través de sus páginas web.

Finalmente, los profesores recomiendan que los reguladores publiquen sus decisiones y expliquen el diagnóstico técnico en que se basan.

20. Por otro lado, casi todos los ejecutivos de las empresas de telecomunicaciones a quienes entrevisté rechazaron como innecesaria la participación del público en el proceso regulatorio, ya que consideran que la Subtel actúa como representante de los ciudadanos. 
21. Irónicamente, las reuniones que Subtel sostuvo con todos estos grupos fueron a puertas cerradas. Ver: El Mercurio online (17 de mayo de 2001): Gobierno Estudia Profunda Reforma a Telecomunicaciones y El Mercurio Online (30 de julio de 2001): Comienza Reforma a Ley de Telecomunicaciones», en www.emol.com. (Sitio visitado el 20 de febrero de 2002.) 